\title{
Identidades ecoculturales en disputa: Una mirada a los conflictos ambientales relacionados con la pesca en México*
}

\begin{abstract}
Resumen
El artículo estudia los conflictos ambientales relacionados con la pesca en Bahía de los Ángeles, Golfo de California, México. Desde el punto de vista teórico se aborda el análisis de las identidades ecoculturales de los pescadores desde la comunicación ecocultural, las teorías postcoloniales y las ideologías ambientales aplicando las metodologías de análisis crítico del discurso y la retórica visual. Los resultados muestran que las identidades están en disputa debido a los procesos de resistencia y adaptación a los cambios materiales y simbólicos relacionados con la privatización, globalización de los mercados y las intervenciones de grupos ambientalistas que hacen presencia en la zona.
\end{abstract}

Mónica Pérez-Marín. Ph.D

Ph.D en Comunicación / Doctora en

Ciencias Sociales.

Universidad de Medellín

Medellín, Colombia

Correo electrónico: moperez@udem. edu.co

perezmoni2000@yahoo.com

다이.org/0000-0002-3562-058X

Google Scholar

Sergio Cruz Hernández.

Ph.D

Doctorado en Ciencias y

Humanidades para el Desarrollo Interdisciplinario.

Universidad Autónoma de Baja California

Baja california, México

Correo electrónico:

checoch@uabc.edu.mx

๑ orcid.org/0000-0001-7383-8902

Google Scholar

\footnotetext{
• El artículo presenta resultados de la investigación "Desequilibrios y asimetrías entre actores implicados en la conservación del Golfo de California" financiado por el Departamento de Posgrado e Investigación de la Universidad Autónoma de Baja California, México. Clave del proyecto 414/1652. El equipo de investigación estuvo conformado por el Dr. Sergio Cruz Hernández (Profesor de UABC), Dra. Mónica Pérez-Marín (Profesora de la Universidad de Medellín, Colombia), Araceli Bernal Calderón (Coordinadora de Comunicación de Pronatura Noroeste -PNO-), Carolina Rodríguez (Asistente de Comunicación en PNO), Nina Alejandra Martínez Arellano (profesora de UABC), Miguel de Jesús Neria Govea. También colaborar en el levantamiento audiovisual Adrián Macías Díaz (realizador audiovisual) y Marco Antonio Meza León (productor y profesor en $\mathrm{UABC}$ ). El equipo de investigación manifiesta su profundo agradecimiento a los habitantes de Bahía de los Ángeles por abrirnos las puertas de sus casas y corazones, y facilitar de este modo el proceso de la investigación.
}

Recibido: junio 25 de 2018 Aprobado: abril 2 de 2019

Palabras clave: arte público, conflictos ambientales, identidades ecoculturales, pesca, procesos simbólicos. 


\section{Contested Eco-cultural Identities: a Look at Environmental Conflicts Related to Fishing in Mexico}

\begin{abstract}
The article studies environmental conflicts related to fishing in Bahia de los Angeles, Gulf of California, Mexico. From the theoretical point of view, the analysis of the eco-cultural identities of fishermen from eco-cultural communication, postcolonial theories and environmental ideologies is addressed applying the critical discourse analysis and visual rhetoric methodologies. The results show that identities are contested, due to the process of resistance and adaptation to the material and symbolic changes related to privatization, globalization of markets and interventions of environmental groups that are present in the area analyzed.
\end{abstract}

Key words:

eco-cultural identities,

environmental conflicts, fishing public arts, symbolic processes. 


\section{Planteamiento del problema}

El Golfo de California, también Ilamado mar de Cortés o mar Bermejo, es la porción de suelo marino y de agua que se encuentra delimitada por la Península de Baja California y por los Estados de Sonora y Sinaloa en México. Posee una extensión de 1.600 km, con anchuras que pueden variar entre los 92 a los 205 km, y una línea de costa de más de 3.000 km; "su superficie marina abarca $247.000 \mathrm{~km}^{2}$ y alcanza profundidades extremas que superan los 3.000 metros" (Tiburcio Pintos \& Cariño Olvera, 2017, p. 9). Cuenta con 898 islas e islotes que representan 50\% del territorio insular mexicano (CONANP, 2000). La diversidad de recursos marinos que posee el golfo lo han convertido en una de las zonas pesqueras más importantes del país ya que "aproximadamente el 80\% de las capturas pesqueras del Pacífico mexicano se generan o al menos se desembarcan en Costas de Baja California, Baja California Sur, Sonora, Sinaloa y Nayarit" (Aragón-Noriega et al., 2017, p. 29).

En los últimos años, el Golfo de California ha estado en la agenda pública de investigadores, científicos, ambientalistas y gobernantes por dos razones centrales: 1) por las prácticas ilegales de pesca en la zona, que se estiman en un $56 \%$ de la pesca nacional; y 2) por las especies marinas en vía de extinción que se capturan de modo accidental como es el caso de la vaquita marina (Phocoena sinus) y la totoaba (Dibble, 2015). Esta situación ha llevado a que en los medios de comunicación circulen posiciones estigmatizantes y románticas sobre las actividades de supervivencia en la zona que ponen en entredicho las prácticas ancestrales de las comunidades de pescadores.

Con relación a las posiciones estigmatizantes, la pesca es presentada por los medios de comunicación, como una práctica desordenada, conflictiva y sin reglas claras entre los pescadores. Esto se confirma desde la revisión de 350 notas publicadas en portales en internet, entre el 1 de abril de 2015 y el 1 de 
diciembre de 2016 (Diario de campo del proyecto de investigación, 2016), donde es posible concluir que en 316 de estas piezas periodísticas, el actor principal de las notas es un externo a las comunidades pesqueras. Por ejemplo el titular de la Secretaría del Medio Ambiente y Recursos Naturales (Semarnat), personajes de World Wildlife Fund (WWF) Greenpeace, investigadores de universidades, etcétera. Solamente en 34 noticias aparecen referenciados actores internos pertenecientes a las comunidades o representantes de alguna organización de pescadores. Un ejemplo de los comentarios que ponen en entredicho las prácticas de los pescadores, está en las declaraciones pronunciadas por Miguel Rivas, coordinador de la campaña para la WWF en México, quien afirma que "ni la prohibición de ciertos métodos de captura, ni los patrullajes en la zona para verificar que efectivamente se esté acatando esta prohibición están siendo efectivos" (ExpocNews, 2016).

Adicionalmente, con el ánimo de justificar el trabajo de conservación que realizan organizaciones ambientalistas en la zona, como Sea Shephered Conservation Society, se insinúa que las comunidades de pescadores de la zona, son las responsables del daño ambiental. En la campaña "Operación Milagro", implementada por esta organización, se realizaron actividades de patrullaje en la zona removiendo redes ilegales. El problema con los mensajes de estas organizaciones que circulan en redes sociales y en los medios de comunicación, es que no especifica quiénes son los responsables de instalar dichas redes, que la mayor parte de las veces pertenecen a piratas marinos que no necesariamente hacen parte de las comunidades de pescadores que habitan la zona. En uno de los videos promovidos por esta organización, se observa que se da aviso a la Marina y a personal de la Procuraduría Federal de Protección al Ambiente (PROFEPA), para que registren las incautaciones de equipo ilegal retirado del mar. Como resultado de sus actividades de gestión en el año 2015, esta organización reporta el rescate de una ballena jorobada, el hallazgo de tres vaquitas marinas muertas atrapadas en redes de enmalle; 
salvamento de siete totoabas, 12 tiburones, 55 rayas; y remoción de 42 redes y 16 palangres ilegales (Resumen Operación Milagro II, 2016).

Con relación a las posiciones románticas, National Geographic Magazine en español, presenta la creación de microempresas y el turismo ecológico como las prácticas deseables a seguir, en la medida en que son un negocio altamente rentable. Un ejemplo de microempresa exitosa, que aparece mencionado en estos reportajes, es la cooperativa pesquera de Abreojos en la cual "la langosta y el abulón se enlatan en una moderna planta de procesamiento y se benefician indirectamente de Asia, lo que maximizan las ganancias (...) las aguas del pueblo están vigiladas por radares, barcos y aviones" (Vance, 2017, p. 24). Con relación al turismo ecológico, se reseña el avistamiento de la Manta birostris o mantarrayas gigantes de cinco metros, localizadas en la Reserva de la biosfera Archipiélago de Revillagigedo a 1000 kilómetros de Manzanillo. Se estima que la población de esta especie en la zona alcanza 350 individuos que generan al año: "14 millones de dólares (...) es decir, cada manta representa un ingreso anual de unos 30.000 dólares si consideramos los 40 años de vida que cada especie tiene en promedio" (Aburto, 2017, p. 41).

El problema con este tipo de representaciones consiste en que, en el espacio público mediático no aparecen documentadas las opiniones y posiciones de los pescadores; tampoco aparecen en dichos reportajes un análisis riguroso de las tensiones y los conflictos que actualmente se viven en la zona. El mensaje que prevalece en este tipo de noticias o reportajes resalta las bondades de la conservación y el turismo "ecológico" de manera idealizada y romántica, desconociendo el contexto histórico y sociocultural en el cual se ha venido realizando la práctica de la pesca. Adicionalmente este discurso no ha ayudado a visibilizar las estructuras de poder que actualmente hacen presencia en la zona y que controlan las actividades relacionadas con la pesca. 
Con el ánimo de conocer de primera mano las posiciones de los pescadores con relación a los conflictos, que se vienen presentando, conectados con la pesca, este proyecto de investigación realizó observaciones directas y entrevistas en Bahía de los Ángeles $(B L A)^{1}$ en el marco del Festival de Arte Público, organizado por la Organización Civil "Cardumen", del 20 al 25 de marzo del 2017, quienes a través de intervenciones artísticas con murales, como proyecto semilla, buscaron generar cambios profundos en la comunidad, promoviendo una pedagogía del diálogo de saberes, transformando el espacio público en una galería urbana que pueda coadyuvar a mejorar la imagen pública que circula en los medios de comunicación (CAP, 2017). Las preguntas de investigación que orientaron nuestra búsqueda fueron las siguientes:

(PI 1): ¿Cuáles son los ejes discursivos relacionados con la identidad que aparecen en los murales del Festival de Arte Público?

(PI 2): ¿Cuál es el contexto de producción de los discursos de los pescadores de BLA en el Golfo de California en relación con las nuevas regulaciones de la pesca y cómo está afectando su identidad?

(PI 3): ¿Cómo se relacionan estos ejes discursivos con las ideologías ambientales?

'De acuerdo con el Censo de Población y Vivienda del 2010, INEGI (2010), en Bahía de los Ángeles se registró una población total de 590 habitantes, de los cuales 313 son hombres y 277 mujeres. La población económica activa fue de 307 y se registró un total de 331 viviendas. 


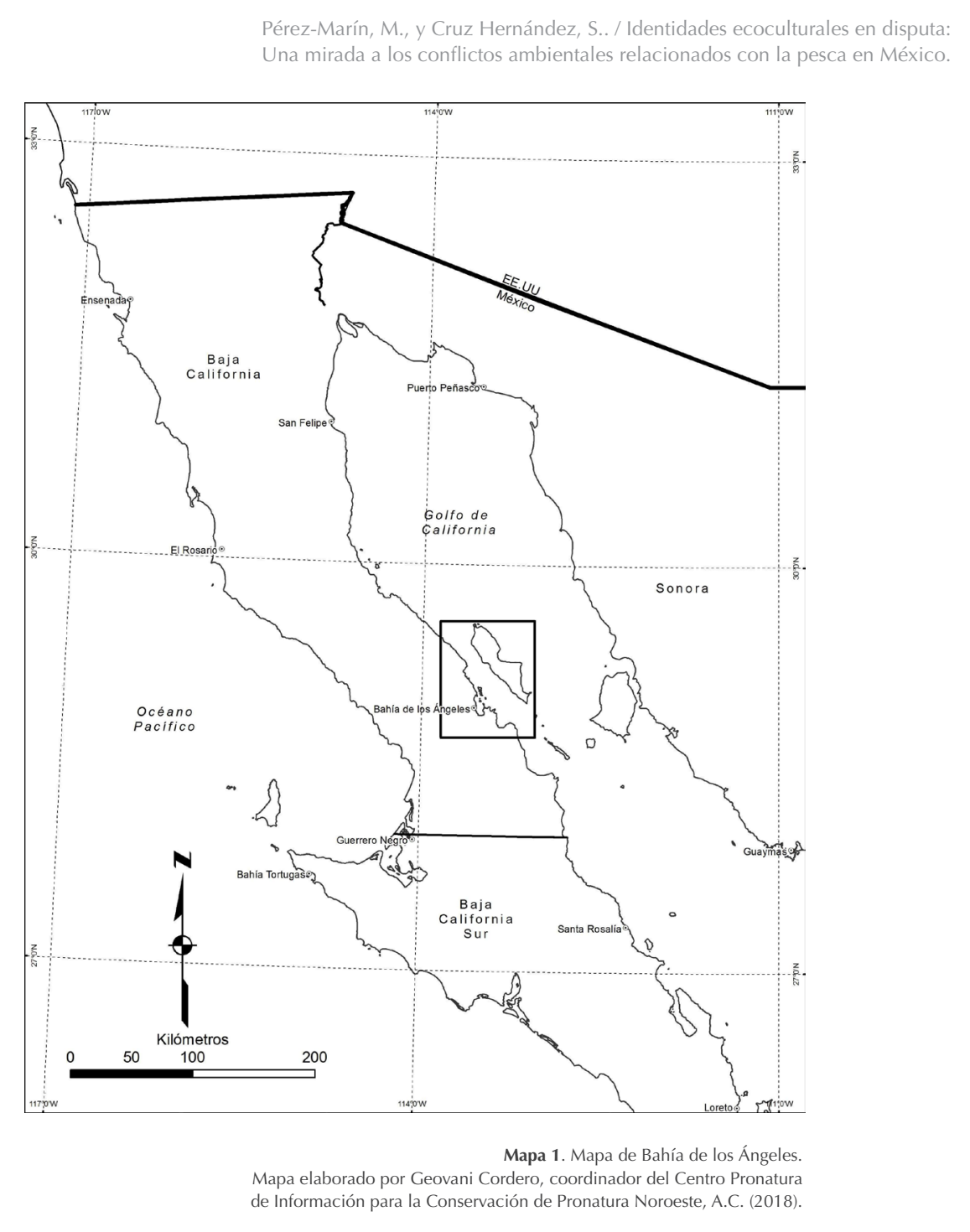




\section{Marco teórico}

\section{Las identidades ecoculturales (IE): una aproximación desde la intersección de la comunicación ecocultural y las teorías postcoloniales}

Con el ánimo de contribuir al vacío en la literatura en relación con el estudio de las identidades desde una perspectiva postcolonial y ecocultural, el presente trabajo propone el concepto de IE que retoma elementos de la fenomenología de Merleau-Ponty (1968, 2006), y de Abram (1996). Por IE entendemos las interacciones materiales y simbólicas (Cruz Hernández, 2015) que se presentan a modo de percepciones, entre seres humanos y no humanos, en un contexto situado e históricamente permeado por condiciones de desigualdad y desequilibrio. Este tipo de interacciones afectan de manera recíproca tanto el estado físico (material) de los individuos que interactúan, como el sentido y el significado de la interacción. En este contexto, este tipo de identidad tiene su origen y se manifiesta a través del cuerpo de los individuos (humanos y no humanos), ya que éste es la puerta de entrada "a lo común, lo intersubjetivo, al campo de la experiencia" (Abram, 1996, p. 44). La fenomenología, corriente filosófica que da base y sustento a nuestro concepto de IE, "no busca dar una explicación del mundo desde afuera, lo que busca es dar voz al mundo desde nuestra experiencia particular y situada, recordándonos nuestra participación (presencia) en el aquí y en el ahora" (Abram, 1996, p. 47).

La investigación le apuesta a pensar las IE desde la intersección de la comunicación ecocultural y las teorías postcoloniales por dos razones fundamentales. La primera, porque nos interesa analizar las IE de los pescadores, más allá de la dicotomía hombre-naturaleza, y en la cual se integran aspectos materiales y discursivos que se manifiestan a través de la emoción, el cuerpo, el color y los sonidos (Abram, 1996). La segunda, porque nos interesa analizar la actividad de la pesca desde una perspectiva situada, histórica, inmersa en 
estructuras de poder, prestando especial atención a las ideologías ambientales que han hecho presencia en la zona de estudio.

La comunicación ecocultural "es un campo de estudio dentro de la disciplina de la Comunicación y, al mismo tiempo, se desempeña como un meta-campo en contacto con otras disciplinas" (Milstein, 2011, p. 344). Su propósito central consiste en pensar "la comunicación y las relaciones de los humanos con el ambiente" $^{\prime \prime}(\mathrm{p}, 344)$. Los investigadores en esta área del conocimiento están "particularmente preocupados por la manera como las personas se expresan o comunican el mundo natural, ya que, este tipo de comunicación tiene efectos a largo plazo en la crisis ambiental que han causado los humanos" (p. 344). Las premisas que orientan la investigación en este campo de estudio son: "1) El medio ambiente incluye procesos materiales y procesos simbólicos y sociales; 2) Las representaciones simbólicas y sociales del medio ambiente incluyen orientaciones "interesadas" hacia su(s) objeto(s); 3) Los contextos socialeseconómicos e ideológicos promueven o reprimen ciertas representaciones sobre el medio ambiente; 4) El sistema dominante de las representaciones sobre el medio ambiente, influye en la toma de decisiones acerca de las señales de deterioro de la salud humana, el clima o el sistema ecológico" (Cox, 2007, pp. 12-14).

Con el ánimo de generar un balance o equilibrio frente a las posiciones antropocéntricas, androcéntricas y etnocéntricas, que han puesto en desventaja a la naturaleza, las aproximaciones ecoculturales defienden la existencia simultánea de "una sociedad humana y de un mundo natural más allá de los humanos" (Milstein, 2011, p. 346), enfatizando una doble representación: una material y una simbólica. Con esta diferenciación, es posible "modificar no solamente la manera como hablamos o nos comunicamos acerca de la naturaleza, sino que también estamos transformando el mundo material". (p. 346). Las implicaciones que se han derivado de pensar el mundo, desde la falsa 
dicotomía excluyente de "los sujetos humanos" y "los objetos no humanos" son: la eliminación de los animales de la madre tierra (Packwood, Bekoff, \& Bexell, 2011; Stibbe, 2012) y la invisibilización del concepto de "lugar" de nuestro horizonte de comprensión (Armstrong, 1995; Boyarin, 1994; Cajete, 1999; Descola, 1996, 2013; Rappaport, 1990). Los académicos que se inscriben bajo esta línea de investigación intentan desafiar y renombrar los presupuestos de las escuelas de pensamiento ambientalistas británicas y norteamericanas de corte ortodoxo, haciendo consciente el lugar y las condiciones sociales e históricas desde las cuales estas teorías fueron formuladas (Boyarin, 1994; Descola, 1996, 2013; Martínez-Alier, 2002, Nixon, 2011a, 2011b); lo cual ha llevado a desviar su atención hacia temas de estudio como son: la justicia ambiental (Evans, 2002; Pezzullo, 2001; Taylor, 2000; Tinker, 1996), las ecoculturas indígenas (Descola, 1996; Valladolid \& Apffel-Marglin, 2001) y las implicaciones de los conceptos de "lugar" (Armstrong, 1995; Boyarin, 1994; Cajete, 1999).

\section{El espectro de las ideologías ambientales}

Siguiendo la línea de estudio de la comunicación ecocultural y los estudios postcoloniales sobre la naturaleza, esta investigación centra su atención en las "ideologías ambientales" ya que permiten develar el carácter fabricado, histórico y cambiante de los significados que hemos atribuido a la naturaleza. De acuerdo con el Diccionario de la Real Academia de la Lengua Española, una ideología es un "conjunto de ideas fundamentales que caracterizan el pensamiento de una persona, colectividad o época, de un movimiento cultural, religioso o político, etc.". En complemento de esta definición, Althusser (2002) afirma que toda ideología involucra un proceso de "representación" mediante el cual se construye una relación imaginaria entre los individuos y sus condiciones reales de existencia. En este contexto, es posible afirmar que las ideas que cada grupo humano tiene sobre la naturaleza son construcciones o representaciones que se han fijado en contextos culturales específicos (Ingold, 1992; Kempton, Bostter \& Hartley, 1996). 
Al aceptar estos presupuestos teóricos, Corbett (2006) propone una re-lectura de las corrientes que han abordado el concepto de naturaleza, resaltando su carácter "fabricado y/o construido", "temporal", "contextual" y "móvil" utilizando el término de ideología. Para lograr dicho propósito, esta autora elabora una "escala" o un "espectro" de las ideologías que han abordado el tema de la naturaleza, de acuerdo con su simpatía o lejanía a lo humano (eje antropocéntrico) o a la naturaleza (eje ecocéntrico o biocéntrico).

ESPECTRO DE IDEOLOGIAS AMBIENTALES

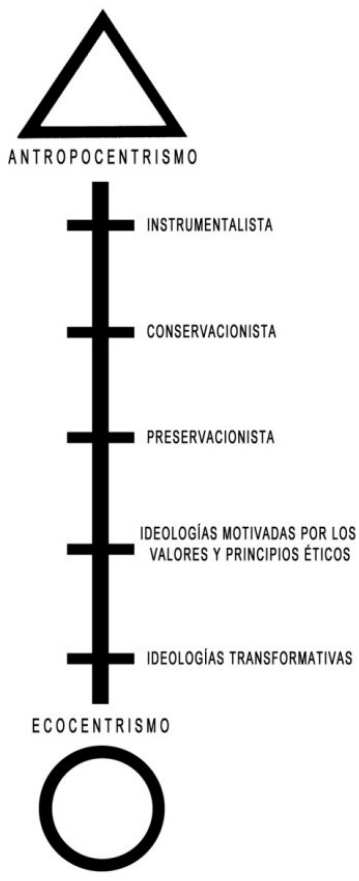

Ilustración 1. Espectro de las ideologías ambientales. Fuente: tomada de Corbett (2016, p. 29). 
El eje antropocéntrico está caracterizado por un triángulo que hace alusión a la jerarquía o estratificación presente en dichas ideologías. El antropocentrismo, defiende el postulado de que "los humanos son superiores y dominan el resto de la creación y el mundo natural está organizado jerárquicamente con los humanos en la cima" (Corbett, 2006, p. 27). Por el contrario, el eje ecocéntrico o biocéntrico, representado por un círculo, defiende el postulado de "una relación de interdependencia no jerárquica, o una interconexión de todo lo que tiene vida" (Corbett, 2006, p. 27). Las ideologías ambientales que aparecen en dicho espectro son: las instrumentalistas, las conservacionistas, las preservacionistas, las ideologías motivadas por valores y principios éticos, y finalmente, las ideologías transformativas (p. 28).

En las ideologías instrumentalistas, todo lo que existe diferente a lo humano es considerado un "recurso", el cual debe estar a disposición para su uso y aprovechamiento, en las ideologías instrumentalistas. La apuesta central de ellas es promover la idea de que "los humanos son las entidades más importantes y dominantes del mundo natural y que todos los recursos existentes se encuentran disponibles para su uso ilimitado" (Corbett, 2006, p. 30). Dos tipos de pensamiento que guardan relación directa con este tipo de ideología se encuentran presentes en las corrientes judeocristiana y helénica, de un lado; y de otro, en el pensamiento de la Ilustración. En la primera tradición, relatos como el mito de Prometeo dan un papel protagónico y central al hombre en contraposición al lugar que se le da a la naturaleza, ya que ésta aparece representada como como un recurso que debe ser dominado gracias a la tecné (Ramírez, 1994, p. 272). En la segunda tradición, el hombre es la medida de todas las cosas en tanto que se enfatiza su superioridad (De Peralta \& Sosa, 1987, p. 92).

Las ideologías conservacionistas se encuentran en un lugar más alejado de las ideologías instrumentistas y más cercanas a las ecocéntricas, continúan 
promoviendo la idea de que: "las entidades no humanas tienen un valor exclusivamente utilitario, esto quiere decir que su valor reside en su potencialidad como recurso para el uso humano" (Corbett, 2006, p. 28). La idea central de estas ideologías consiste en usar o manipular mediante el conocimiento científico y técnico los recursos naturales. La atmósfera de la época entre 1890-1920, época en la que se crea y generalizan estas miradas de la naturaleza buscaban un "crecimiento económico, sacando el máximo partido a los recursos naturales, especialmente los renovables" (Ramos, 2002, p. 32). Un ejemplo de una ideología conservacionista se encuentra en el desarrollo sustentable. Este tipo de pensamiento, al tiempo que intenta asegurar a las futuras generaciones, el agua, la tierra y otros recursos naturales, también se interesa por su aprovechamiento económico (Corbett, 2006; Barbier, 2005). En conclusión, un conservacionista es, de alguna manera, un pensador conservador, quien defiende o practica el uso sostenible y cuidadoso de los recursos naturales asegurándolos para las futuras generaciones (Pinchot, 1910, pp. 48-80).

El tercer lugar lo ocupan las ideologías preservacionistas. Los postulados centrales de estas ideologías presentan un avance con relación a las ideologías conservacionistas, ya que atribuyen ciertos elementos ecocéntricos, como la idea de otorgar un valor intrínseco a la naturaleza -que puede ser ecológico, científico o religioso-, diferentes a un valor estrictamente económico (Corbett, 2006). El valor científico está asociado al valor de la naturaleza como fuente o banco de datos, genes o valor genético aún no descubiertos. El valor estético está asociado a las posibilidades de goce, disfrute y belleza que genera la naturaleza en el ser humano, restaurando los estados de equilibrio. El valor religioso resalta la condición de "templo" o "catedral" de la naturaleza, como el lugar original en el que reposa lo sagrado (Corbett, 2006). Un ejemplo de las ideologías conservacionistas se encuentra en el concepto de parque nacional natural - $\mathrm{PNN}-$, que surgió en los Estados Unidos de América, 
durante la expansión del lejano oeste. En 1872, el Congreso de los Estados Unidos utilizó por primera vez la denominación parque nacional para reservar y separar cualquier impacto de colonización y de ocupación humana de las 899.139 hectáreas, en el curso superior del río Yellowstone, Wyoming. Con este nuevo concepto o categoría, además de complacer el deseo de las élites de disfrutar de espacios para el ocio y el descanso, se buscó disponer de un nuevo dispositivo de control, una nueva "excusa" para tener el dominio y garantizar el progreso económico de la nación. Con el argumento de proteger y preservar algunos "escenarios superlativos, flora y fauna de trascendencia nacional para que el público en general lo disfrutara y pudiera beneficiarse si era puesto bajo control público" (Rummenhoeller, 1995, p. 66), se inicia una confrontación inédita, con muchas aristas, que dio origen a algunos de los conflictos ambientales, como el que se aborda en esta investigación.

Las consecuencias de esta mirada conservacionista de la naturaleza, controladas bajo las figuras de área protegida, área de conservación o PNN, se convierte en una figura arbitraria y polémica porque "impone desde afuera normas, desconoce realidades y derechos, sobrestima los instrumentos y subestima la capacidad de resistencia del actor local, o su posibilidad de emerger como agente de cambio" (Andrade, 2009, p. 52). Adicionalmente, esta mirada sobre el territorio continúa perpetuando de manera matizada la relación dicotómica hombre-naturaleza o naturaleza-cultura (Pérez-Marín, 2012, 2015a, 2015b 2016a, 2016b, 2017), generando como resultado "la construcción de una identidad imperialista, por intermedio del pensamiento del "otro" siendo codificado, o bien como "naturaleza", o como "negros", o como "salvajes" y así sucesivamente" (Palacio, 2001, pp. 54-55).

En una cuarta posición, se encuentran las ideologías motivadas por los valores y principios éticos; estas ideologías incorporan una dimensión moral y ética al comportamiento humano, bajo el postulado de que las entidades no humanas 
tienen derecho a existir, al tiempo que los seres humanos tenemos deberes frente a ellas. En este sentido, "la naturaleza pasa de ser objeto de derechos asignados por los humanos, a ser ella misma sujeto de derechos y por lo tanto, posee valores intrínsecos" (Gudynas, 2009, p. 38). La idea central de estas ideologías es que todos los seres vivos, incluyendo los no-humanos, "compartimos una comunidad biótica [que es mucho más ecocéntrica que el nivel anterior], pero continúa cercana a la jerarquía tradicional en la cual los humanos se encuentran en la cima" (Corbett, 2006, p. 37). Un ejemplo de esta ideología es la Constitución actual de Ecuador en la cual se incluyen por primera vez conceptos como "derechos de la naturaleza" y "derecho a su restauración", con los cuales "se genera una nueva articulación con los saberes tradicionales, al referirse, tanto a la naturaleza como la Pachamama (...) basado en la nueva vida [sumak kawsay: buen vivir] y en nuevas estrategias de desarrollo" (Gudynas, 2009, p. 34).

El último lugar lo ocupan las ideologías transformativas. Estas ideologías prestan especial atención a la manera cómo nos comunicamos y cómo nombramos la naturaleza, con el ánimo de generar un cambio que modifique, no solamente las prácticas comunicativas con las cuales nos referimos a ella, sino también que genere un verdadero cambio social. Algunas de las ideologías ambientales que se inscriben dentro de esta corriente de pensamiento son: ecología profunda, ecología social, el eco-feminismo, las ideologías de los nativos americanos, las tradiciones orientales. Para Cox, (2007); Milstein, (2008); Milstein y Dickinson, (2012); Milstein y Krolokke, (2012); Peterson, Peterson y Peterson, (2007); y Rogers (1998), el concepto de naturaleza no solamente ha estado influenciado por un mundo con mirada antropocéntrica (ser humano como centro de todas las cosas), sino también por el androcentrismo (la visión masculina como principal) especialmente estudiado por las feministas norteamericanas y por el eurocentrismo (visión europea como principal en la civilización) estudiado por las teorías postcoloniales. 


\section{Metodología}

El trabajo de campo se realizó en tres momentos en BLA. El primero del 4 al 6 de febrero del 2017 en la cual se realizaron las primeras observaciones y entrevistas semiestructuradas a las comunidades, se entrevistó un funcionario de la Comisión Nacional de Áreas Naturales Protegidas (CONANP), al director de preparatoria de CECYTE y un pescador de pulpo. El segundo momento tuvo lugar del 6 al 8 de marzo del 2017, periodo de tiempo en el cual se realizó la primera salida a la pesca de pulpo con el pescador y su ayudante, lo que permitió conocer mejor la actividad que realizan y que los pescadores tuvieran mayor confianza con el entrevistador. Y finalmente el tercer momento, del 19 al 25 de marzo del 2017 en la cual se llevó a cabo el festival de arte público CAP en BLA. En el evento participaron 11 artistas principalmente mexicanos, un canadiense y un español. De todas las actividades realizadas se dejó un registro que puede ser consultado online ${ }^{2}$.

El objetivo central del CAP consiste en generar cambios profundos en la vida social y cultural de la comunidad, a través del arte, generar un diálogo en relación con la problemática ambiental, convirtiendo la ciudad (en este caso el pueblo) en una galería urbana. Adicionalmente, les interesa afianzar la identidad de las comunidades ya que ésta y muchas otras comunidades de la península de Baja California, se han estigmatizado fuertemente por los medios de comunicación. Algunas de estas poblaciones, se encuentran conformadas por personas jóvenes, recién llegadas o migrantes, lo que ha ocasionado desarraigo y falta de sentido de pertenencia. El CAP se encuentra liderado por una organización de la sociedad civil Ilamada Cardumen, la cual cuenta con el apoyo de otras organizaciones tales como Pro Esteros, A.C., Pronatura Noroeste, A.C. y también de la Facultad de Ciencias Administrativas y Sociales de la Universidad Autónoma de Baja California.

\footnotetext{
${ }^{2}$ Para ver el documental de la investigación se sugiere ver: https://vimeo.com/325571105

Para ver las fotografías de los murales se sugiere visitar el sitio: https://www.facebook.com/CAP.Bahia/?__tn__=\%2Cd\%2CP_ R\&eid=ARAamR8SmU6o5qcvN8DFBLT-aUAuH_kXZrGAEah51VdDAtXtgJYRgV_ERfAbO0zhDn9nb2IMk6a4Tioj
} 
Para el análisis de la información se utilizó la metodología de análisis crítico del discurso (ACD), particularmente el análisis tridimensional de Fairclough (1989, 1992, 1995) que contempla tres niveles de análisis: el textual, el de las prácticas comunicativas y el de las prácticas socioculturales. El primer nivel, el textual, se refiere a los núcleos discursivos o regímenes de representación (Rojas, 2002; Escobar, 2012; Pérez-Marín, 2016a, 2016b, 2017) que circulan y han circulado en la comunidad de BLA con relación a la pesca. El segundo nivel, el de las prácticas comunicativas, explora el contexto de producción de dichos discursos desde una perspectiva institucional. Y finalmente el tercer nivel, el de las prácticas socioculturales, trata de hacer un análisis más profundo y macro-contextual del fenómeno estudiando a la luz de las ideologías que han configurado las representaciones que hemos tenido y actualmente tenemos sobre la naturaleza, el medio ambiente y en este caso particular sobre la pesca.

Como técnica complementaria para analizar el primer nivel, el textual, en nuestro caso los murales del CAP, se utilizó la metodología de retórica visual (RV) de Foss (2004), ya que esta técnica analiza la función de la imagen para complementar y conectar directamente con las prácticas comunicativas y prácticas socioculturales de ACD. De acuerdo con Foss (2004), esta técnica de investigación permite analizar "el proceso simbólico desde el cual las representaciones visuales realizan el proceso de comunicación" (p. 304). Los tres niveles de análisis que propone la RV son: naturaleza, función y evaluación (Foss, 2004).

El primer nivel, la naturaleza, busca explorar "elementos como metáforas, argumentos, ethos, evidencia, narrativa y movimiento" (Foss, 2004, p. 308); con relación a los elementos visuales se recomienda analizar "el color, espacio, la textura y la vectorialidad" (Foss, 2004, p. 308). El segundo nivel, la función explora la acción que la foto o el objeto visual está comunicando; algunas funciones de los artefactos visuales "pueden ir desde el homenaje a ciertos 
individuos hasta la creación de sentimientos de calidez y comodidad para alentar a los espectadores a explorar llimitaciones autoimpuestas" (Foss, 2004, p. 308); en este sentido la función se encuentra directamente relacionada con el tipo de emoción que la imagen comunica. La tercera dimensión, la evaluación busca juzgar la imagen desde el propósito o la intencionalidad con la que fue construida, es decir si la imagen logra cumplir el propósito para la cual se creó (Foss, 2004, p. 309).

\section{Resultados}

A continuación presentamos los resultados encontrados respondiendo a cada una de las preguntas de investigación:

\section{1. ¿Cuáles son los ejes discursivos relacionados con la identidad que aparecen en los murales del Festival de Arte Público?}

Después de realizar el anális de los los 11 murales aplicando la metodología de VR (Foss, 2004) podemos concluir que con relación con el primer nivel de análisis, naturaleza, los murales se pueden clasificar del siguiente modo: 1) Los que presentan un solo elemento como protagonista del mural: "Garza" de Diana Bama, "Ballena" de Emerson Baldera, "Vaquita Marina" de Himed y "Tiburón ballena" de Mocre. 2) Los que enfatizan la diversidad y variedad marina retomando como protagonistas varias especies: "Personajes de BLA" de Emerson Baldera, "El rey" de Gangsby, "Mural Vitral" de Carlos Soto y "Corales" de Esther Gámez. 3) Los que atribuyen a la naturaleza aspectos humanos: "Mujer del mar" de Mónica Loya y "Cerros e islas coloreados por el atardecer" de Cyrielle Tremblay. Y 4) Los que atribuyen a la naturaleza un aspecto sagrado: "Corazón de mar y tierra" de Dherzú Uzala y "Niño Kiliwa" de JM. 
Desde el aspecto de la función en la cual se estudia la emoción que comunican los murales, se encontraron los siguientes elementos: energía, vida, movimiento; riqueza y variedad de los recursos marinos; el carácter sagrado de la naturaleza; la capacidad de ver y sentir de la naturaleza; la belleza y armonía del paisaje marino; la pasividad o desconexión de la mujer (o los humanos en general) con lo que está sucediendo alrededor; la integración e interconexión de todas las especies vivas, incluyendo los humanos; el hombre y las especies marinas comparten un mismo espacio.

Finalmente, con relación a la evaluación, en la cual se analizan los mensajes conectándolos con la problemática ambiental y con las identidades, encontramos que la mayor parte de los murales presentan una mirada de la naturaleza de corte conservacionista o preservacionista, otorgando un valor estético o científico a los recuros naturales, lo que confirma que las identidades se siguen configurando, la mayor parte de las veces, desde posiciones antropocéntricas (Corbett, 2006). Se observa un ligero avance hacia posiciones éticas y de valores con relación a la naturaleza en los murales: "Mujer del Mar" de Mónica Loya y "Cerros e islas coloreados por el atardecer" de Cyrielle Tremblay, ya que estos nos permiten hacer consciente la responsabilidad que tenemos frente a la naturaleza y sus recursos, y nos interrogan sobre la insensibilidad y ceguera que estamos presentando frente a la problemática ambiental. En este sentido la identidad que aparece reflejada en estos murales comienza a resistir y a oponerse a la falsa dicotomía hombre-naturaleza que caracteriza al pensamiento moderno, eurocéntrico y antropocéntrico, desde el cual se legitima el uso y explotación de la naturaleza.

Otros dos murales que cuestionan esta dicotomía y el pensamiento moderno y que se aproximan a posiciones transformativas de la naturaleza (Corbett, 2006) son "Corazón de mar y tierra" de Dherzú Uzala y "Niño Killwa" de JM. En el primero, se enfatiza el carácter sagrado de la naturaleza al representar un 
corazón que tiene tantos elementos humanos como marinos y que se encuentra custodiado por un viejo sabio bajo la forma de un cactus. El segundo mural relata la historia de la etnia Cochimí, antiguos habitantes de la Baja California; en el centro aparece un niño que cuida de la naturaleza y juega con borregos cimarrones, lobos marinos, gavilanes y tortugas; especies significativas para la cultura Cochimí porque representan a los guardianes del cielo, el mar y la tierra, además de las estrellas, las cuáles figuraban como guías en sus travesías. El tipo de identidades que se expresa en este tipo de murales hace un Ilamado de atención sobre el conocimiento, sabiduría y respeto hacia la naturaleza de las primeras comunidades que han habitado el territorio: la Maya y la cultura Cochimí.

\section{2. ¿Cuál es el contexto de producción de los discursos de los pescadores de BLA en el Golfo de California con relación a las nuevas regulaciones de la pesca y cómo está afectando su identidad?}

El contexto de producción de los discursos desde el cual se ha configurado la actividad pesquera en BLA ha sufrido grandes transformaciones debido a los cambios de las políticas públicas que han regulado la actividad pesquera y a los cambios sufridos en la oferta de este recurso relacionada con los temas de la sobreexplotación, el cambio climático y los intereses de las multinacionales. Una mirada a la política pública pesquera de México nos permite concluir que la figura de "Sociedad Cooperativa Pesquera" ocupó desde comienzos del siglo XX un lugar central y protagónico, enfatizando el mejoramiento social y económico de las comunidades de pescadores; lo cual otorgó a este grupo social un gran reconocimiento social y un cierto grado de "distinción" (Bourdieu, 1988) en el ámbito local y nacional.

En la etapa correspondiente al periodo 1986-1991, denominada la planeación pesquera, se logra un gran avance constitucional al introducir por primera vez 
los conceptos de los "derechos de las comunidades" sobre la explotación de los recursos marinos pertenecientes a las cooperativa pesqueras, los ejidos y las comunidades agrarias (Valenzuela Reyes, 2012). En este sentido, también se introdujo el término "responsabilidad social", garantizando el derecho de las comunidades pesqueras a completar y mejorar sus ingresos.

Con la Ley de Pesca de 1992, observamos un cambio drástico en la política pública que promovía y fomentaba la figura de cooperativa pesquera, ya que se elimina la exclusividad de las cooperativas en la captura de especies reservadas, arguyendo que estas necesitan altas inversiones que superan su capacidad, por lo cual deben aliarse con otros agentes económicos (Valenzuela Reyes, 2012). En este momento se abre la puerta para los intereses privados y de las multinacionales. De acuerdo con Greenpeace:

\footnotetext{
Las pesquerías en México están sobrexplotadas, por lo que en dos décadas han caído en alrededor de 30 por ciento [...] esto ocurre debido a que en el país no existe una política clara y estratégica dirigida al sector [...] de acuerdo con los datos más recientes, en el Golfo de México las capturas totales tienen una tendencia a la baja; hasta ahora han descendido 20 por ciento, luego de que en 1987 alcanzaron su máximo histórico. (La Jornada, 24 de febrero de 2010)
}

Si bien la Ley General de Pesca y Acuacultura Sustentable del 24 de julio de 2007 sigue resaltando ciertos aspectos de caracter cooperativo, como los siguientes: 1) la perspectiva sostenible en la cual se integren y concilien factores económicos, sociales y ambientales; 2) el impulso regional equilibrado y equitativo; 3) la participación y consenso de las comunidades en el aprovechamiento integral y sustentable de los recursos naturales. En la práctica la realidad es otra, ya que con el incremento de las NOM (normas oficiales mexicanas) que regulan la actividad pesquera, surgen nuevos problemas en las comunidades de pescadores. De acuerdo al trabajo de campo realizado en el marco del Festival de Arte Público CAP, los problemas identificados que están afectando la construcción de la identidad de los pescadores son: el abandono 
del Estado: la ausencia de empleo, de actividades lúdicas para los jóvenes y la drogadicción.

Sobre la ausencia del Estado en la zona, el exdirector Domingo de Jesús Zatarain González de la Reserva de la Biosfera de BLA Bahía, Canales de Ballenas y Salsipuedes, afirma que:

\begin{abstract}
A pesar de que es una ubicación privilegiada de recursos naturales, para los pobladores es un reto vivir en condiciones de aislamiento, la energía eléctrica es mucho más cara, el agua es escasa, abastecerse de los insumos primordiales para la vida, la gente se va adaptando; hay vacíos institucionales para acercar los servicios, aquí no hay servicio de transporte público, no hay servicio bancario ni cajeros automáticos, no hay como retirar dinero, no hay apartado postal, no hay señal de celular, algunos tienen telefonía microondas, esto los margina a los pobladores; y pueden ser presa de las drogas y el alcohol que se ve muy acentuado. (Diario de Campo BLA, 2017)
\end{abstract}

Con relación a la ausencia de actividades lúdicas para los jóvenes y la drogadicción, el lider local y pescador, José Arce Smith, conocido como el "Güero", afirma que es necesario crear programas que amplien la esfera pública de los jóvenes de la zona ya que sus interacciones no se pueden limitar a la vida familiar y escolar. En ese sentido, el Güero decidió crear un taller de fotografía con los niños y jóvenes de la zona con el ánimo de:

Alejarlos un poco de la drogadicción, de la vagancia, del alcoholismo. Empecé con la fotografía [...] empezamos a ver la posibilidad de armar un grupo de Bahía, así fue que empezó". "Involucrarlos en la fotografía para evitar que caigan, es el problema que hemos tenido, yo todavía la he vivido en esta zona, me he dado cuenta de como niños y jóvenes se inclinan por la drogadiccion y el alcoholismo, rescatarlos un poco [...] enseñarles un mejor camino. Hablar con ellos, cada salida al campo se les explica. (Bahía de los Ángeles) Es una comunidad muy alejada de las grandes ciudades, falta autoridad, la presencia de autoridades se debe la cantidad de droga que se maneja en estos pueblitos. El problema es que la droga la venden aquí, muchos no tienen la posiblidad de salir, terminan su escuela y terminan pescando o trabajando en tierra estando aquí caen en el alcoholismo o la drogadicción y lo que estamos viendo es una opción de que alguno de ellos le nazca o le llame la atención. (Diario de Campo BLA, 2017) 
A pesar de que en BLA, existe presencia de organizaciones de la sociedad civil — ONG - principalmente de corte ambientalistas, el trabajo que vienen realizando no involucra el estudio de dimensiones sociales, históricas y culturales, lo cual nos permite afirmar que dichas prácticas siguen cimentadas bajo la dicotomía hombre/naturaleza, perspectiva desde la cual la naturaleza es un recurso para el beneficio del hombre, es decir, las estrategias de intervención continúan ancladas en ideologías preservacionistas o conservacionistas.

Sobre las dificultades que se vienen presentando de la planeación sustentable en los sectores rurales como BLA, se concluye que:

La planeación comunitaria realizada para Bahía de Los Ángeles, ha servido de poco para Ilevar a cabo las estrategias planteadas para el desarrollo económico de la pesca, el turismo y el desarrollo urbano del asentamiento. Lo que se ha promovido más bien son una serie de acciones atomizadas y desarticuladas que al momento de querer juntarlas no ofrecen un proyecto comunitario de desarrollo con visión a futuro para la localidad y su región. (Rojas Caldelas, 2011, p. 141)

\section{3. ¿Cómo se relacionan estos ejes discursivos con las ideologías ambientales?}

Con relación al primer nivel de análisis de los murales, los ejes discursivos o el nivel textual (Fairclough, 1989, 1992, 1995), en la muestra analizada predominan las ideologías conservacionista o preservacionista. Los ocho murales que reproducen estas ideologías son: "Garza", "Ballena", "Vaquita Marina", "Tiburón ballena", "Personajes de BLA", "El rey", "Mural Vitral" y "Corales". Los murales que expresan una relación directa con las ideologías ética y de valores son: "Mujer del Mar" y "Cerros e islas coloreados por el atardecer". Finalmente los murales que promueven las ideologías transformativas son: "Corazón de mar y tierra" y "Niño Kiliwa".

El predominio de las ideologías conservacionistas y preservacionistas en el Festival de Arte Público confirman que éstas siguen jugando un rol central en 
la construcción de las identidades de los habitantes de BLA. Lo problemático de estas ideologías es que siguen reproduciendo una mirada dicotómica del mundo, hombre/naturaleza, en la cual los humanos quedan por fuera del mundo de la naturaleza y asumen dos roles antagónicos: 1) En el primer rol los humanos, en este caso los pescadores, como los responsables del deterioro ambiental y de la muerte de las especies en vía de extinción; como lo han relatado algunos medios de comunicación de la zona, en los cuales los pescadores quedan estigmatizados como los responsables; y 2) En el segundo rol, los humanos, en este caso los científicos, son representados como los salvadores, ya que tienen el conocimiento y las técnicas para detener y preveer el deterioro ambiental.

Otro aspecto controvertido de estas ideologías radica en que la naturaleza se asume como un "objeto" de estudio, sujeto a los cánones de la medición y el control; en otros casos se asume como un "recurso" que está al servicio del hombre para generar desarrollo y progreso, lo cual la mayoría de las veces se traduce en generar ganancias y rentabilidad económica. En ambos casos finalmente, a la naturaleza se la despoja de su propio Ser, del derecho a existir por ella misma, ya que el hombre se atribuye el derecho de cambiarla y modificarla a su antojo, utilizando los argumentos de desarrollo, modernidad, progreso, educación, goce o disfute, etc.

Con relación a los murales que se asocian con las ideologías ética y de valores, "Mujer del Mar" y "Cerros e islas coloreados por el atardecer", se observa un gran avance al reconocer en la naturaleza cierta autonomía con relación a los humanos, y resaltar en los murales algunas sensaciones o atributos de la naturaleza como son: la capacidad de ver, en la cual se puede estar muy atento y con los ojos muy abiertos como es el caso de "Cerros e islas coloreados por el atardecer" o muy dormido sin deseos de despertar como es el caso de "Mujer del Mar". En cuanto a los murales que se conectan con las ideologías 
tranformativas "Corazón de mar y tierra" y "Niño Kiliwa", es importante resaltar que representan la naturaleza desde dos tradiciones en las cuales no se evidencia la dicotomía hombre/naturaleza; todo lo contrario, se integran y complementan. Estas tradiciones son: la Maya y la Cochimí.

Con relación al segundo nivel de análisis, las prácticas comunicativas (Fairclough, 1989, 1992, 1995), en el cual se analizan las políticas públicas desde las cuales se ha institucionalizado y puesto en práctica la actividad pesquera, encontramos que la figura de "cooperativa de pescadores" ha sido un elemento central de la política pública pesquera al jugar un papel central en la configuración de la identidad de los pescadores de la zona. A pesar de ello, esta figura se ha venido debilitando en los últimos años, especialmente desde el periodo de la "Adopción de la visión pesquera mundial" (1992-2000), debido a los procesos de globalización y privatización que reducen cada vez más la responsabilidad del Estado.

En este contexto de mercados globales e intereses privados, algunos autores sugieren conectar los intereses locales, con los regionales, nacionales y globales, mediante la capacitación de líderes social que participen en la toma de decisiones a través de los comités consultivos para el manejo pesquero, ya que de acuerdo con Rodrigo Elizarrarás, Director de Política Pública para EDF de México:

Los Comités Consultivos de Manejo promueven una gobernanza efectiva, transparente y representativa; han mostrado ser eficientes en México y otros países como Belice y Chile, ya que operan a nivel local, pero con apoyo estatal y federal, lo cual permite la formalización y vinculación de los acuerdos alcanzados (Suárez, 2017).

En este mismo sentido, José Luis Carrillo Galaz, presidente de la Federación Regional de Sociedades Cooperativas de la Industria Pesquera de Centro y Ponente del Estado de Yucatán afirmó que "por la importante contribución del 
sector social a la pesca y las muchas propuestas que tenemos para resolver los problemas del sector, tenemos que estar en la mesa donde se toman las decisiones" (Suárez, 2017).

\section{Conclusiones}

Las IE de los pescadores en BLA están en permanente cambio y transformación, debido a los cambios de las condiciones materiales y simbólicas que actualmente se vienen presentando en la zona. Estas identidades están en disputa ya que se encuentran en proceso de resistencia y/o adaptación a los cambios materiales y simbólicos relacionados con los procesos de privatización, la globalización de los mercados y las intervenciones de grupos ambientalistas que centran sus estrategias de trabajo desde ideologías conservacionistas y preservacionistas que dejan por fuera el componente social, histórico y cultural de esta comunidad de pescadores.

Las condiciones materiales que están afectando la configuración de dichas identidades están relacionadas, de un lado, con la escasez del recurso pesquero y la necesidad de conservar las especies en vía de extinción; de otro lado, con una política pública débil que ha dejado la puerta abierta para el ingreso de capitales internacionales, dejando en desventaja a la figura de cooperativa de pescadores, que desde la constituyente de 1917 en el artículo 28 jugó un papel central. Adicionalmente, se observa un abandono y ausencia del Estado en la zona, que se manifiesta en la falta de empleo, falta de oportunidades lúdicas y de esparcimiento para jóvenes y en el aumento de las tasas de drogadicción.

Con relación a las condiciones simbólicas, observamos que las ideologías ambientales que se siguen fomentando y reproduciendo, de manera hegemónica en la comunidad de pescadores de BLA, son las conservacionistas y preservacionistas. El problema con este tipo de ideologías consiste en que 
ellas siguen promoviendo la dicotomía hombre/naturaleza, privilegiando el uso y la explotación de la naturaleza con fines económicos o científicos - dentro de los cuales se incluyen los argumentos relacionados con la conservación-. Frente a esta situación se recomienda fortalecer las comunidades de base para recuperar y defender la figura cooperativa de pescadores, no solamente a nivel jurídico, sino también simbólico retomando elementos de las ideologías transformativas Maya y Cochimí.

\section{Referencias}

Abram, D. (1996). The spell of the sensuous: perception and language in a more-than human world. New York: Pantheon Books.

Aburto, O. (2017). Islas Revillagigedo. Cómo salvar el paraíso. National Geographic en Español, 41 (3), 38-43.

Althusser, L. (2002). La filosofía como arma de la revolución. México: Siglo XXI.

Andrade, G.I. (2009). ¿El fin de la frontera? Reflexiones desde el caso colombiano para una nueva construcción social de la naturaleza protegida. Revista de Estudios Sociales, (32), pp. 48-58.

Aragón-Noriega, E.A., Castillo-Vargasmachuca, S.G., Ponce-Palafox, J.T., CruzVásquez, R., Rodríguez-Domínguez, G. y Pérez-González, R. (2017). Distribución potencial de almeja de sifón Panopea globosa del Golfo de California en un escenario de cambio climático. Acta Universitaria, 27 (3), 28-35. http://dx.doi.org/10.15174/au.2017.1245

Armstrong, J. (1995). Keepers of the Earth. En: T. Roszak, M. Gomes \& D. Kanner (Eds.), Ecopsychology: Restoring the Earth, Healing the Mind (pp. 316-324). San Francisco: Sierra Club Books. 
Barbier, E. (2005). Natural Resources and Economic Development. Cambridge: Cambridge University Press.

Bourdieu, P. (1988). La distinción. Criterios y bases sociales del gusto. Madrid: Editorial Taurus.

Boyarin, J. (1994). Space, Time, and the Politics of Memory, (pp. 1-37). En: J. Boyarin (Ed.) Remapping Memory. The Politics of TimeSpace. Minneapolis: University of Minnesota Press.

Cajete, G. (1999). Reclaiming biophilia: Lessons from indigenous peoples. En: G.A.S.D.R. Williams (Eds.), Ecological education in action: On weaving education, culture, and the environment. (pp. 189-206). Albany, NY: State University of New York Press.

CAP. (2017). Festival de Comunidad y Arte Público. Bahía de los Ángeles, México.

CONANP (2000). Comisión Nacional de Áreas Naturales Protegidas. Programa de Manejo Área de Protección de Flora y Fauna Islas del Golfo de California, México. Recuperado de http://islasgc.conanp.gob.mx/archivos/ISLAS\%20 GOLFO\%20\%20F.pdf.

Corbett, J.B. (2006). A spectrum of environmental ideologies. En: Communicating Nature: How We Create and Understand Environmental Messages (pp. 2656). Washington, DC: Island Press.

Cox, R. (2007). Nature's 'Crisis Disciplines': Does environmental communication have an ethical duty? Environmental Communication: A Journal of Culture and Nature, 1, 5-20. https://doi.org/10.1080/17524030701333948

Cruz Hernández, S. (2015). Los recolectores Yumanos del piñón: Identidades y representaciones de la relación con la naturaleza en una práctica transgeneracional interpretada desde la perspectiva simbólica (tesis de 
doctorado). Disponible en: https://drive.google.com/open?id=0B0Rm65_ SYHPhZmlCN1FpX3NrZzA

De Peralta y Sosa, J.M. (1987). La ilustración en Europa y los criterios sobre el mundo clásico. Norba, Revista de Historia, (8, 9), 87-103.

Descola, P. (1996). The Society of Nature. A Native Ecology in Amazonia. Cambridge: Cambridge University Press.

Descola, P. (2013). Anthropological Dualism. (pp. 27-54). En: Ph. Descola (Ed.). The Ecology of Others. Chicago: Prickly Paradigm Press.

Diario de Campo del Proyecto de Investigación. (2016). Seguimiento al cubrimiento noticioso de las noticias publicadas en internet con relación a las comunidades pesqueras. Proyecto de Investigación "Desequilibrios y asimetrías entre actores implicados en la conservación del Golfo de California" financiado por el Departamento de Posgrado e Investigación de la Universidad Autónoma de Baja California.

Diario de Campo BLA. (2017). Visitas a BLA en 2017. Proyecto de Investigación "Desequilibrios y asimetrías entre actores implicados en la conservación del Golfo de California" financiado por el Departamento de Posgrado e Investigación de la Universidad Autónoma de Baja California.

Dibble, S. (2015, December 16). Mexico's efforts to protect vaquita lauded. The San Diego Union-Tribune. California.

Escobar, A. (2012). Encountering development: The making and unmaking of the Third World. Princeton, NJ: Princeton University Press.

Evans, M. (2002). Nature and environmental Justice. En: J. Adamson, M. Evans, \& R. Stein (Eds.), The Environmental Justice Reader: Politics, Poetics, and Pedagogy (p. 181-193). Tucson: University of Arizona Press. 
ExpocNews (2016). La vaquita marina hoy está más cerca de la extinción. 2016, 18 de abril. Recuperado de https:/www.expoknews.com/la-vaquitamarina-hoy-esta-mas-cerca-de-la-extincion/

Fairclough, N.L. (1989). Language and power. London: Longman.

Fairclough, N. (1992). Discourse and social change. Malden, MA: Blackwell Publishing.

Fairclough, N. (1995). Media Discourse. New York: Hodder.

Foss, S.K. (2004). Framing the Study of Visual Rhetoric: Toward a Transformation of Rhetorical Theory. In C.A. Hill, M. Helmers (Eds.), Defining visual rhetorics (pp. 303-313). Mahwah, NJ US: Lawrence Erlbaum Associates Publishers.

Gudynas, E. (2009). La ecología política del giro biocéntrico en la nueva constitución de Ecuador. Revista de Estudios Sociales, (32), 34-47.

INEGI. (2010). Censo de Población y Vivienda 2010. Recuperado de http:// www.beta.inegi.org.mx/proyectos/ccpv/2010/

Ingold, T. (1992). Culture and the Perception of the Environment. (pp. 39-56). En: E. Croll \& D. Parkin (Eds.) Bush Base: Forest Farm. Culture, Environment and Development. London: Routledge.

Kempton, W., Boster, J. \& Hartley, J. (1996). Cultural Models of Nature. (pp.3962). En: W. Kempton, J.S. Boster \& J.A. Hartley (Eds.). Environmental Values in American Culture. Cambridge: Massachusetts Institute of Technology.

La Jornada. (2010, 24 de febrero). Sobrexplotación en las pesquerías de México. Periódico La Jornada, p. 44. Recuperado de http://www.jornada.unam. mx/2010/02/24/sociedad/044n3soc

Martínez-Alier, J. (2002). The Enviromentalism of the Poor. A Study of Ecological Conflicts and Valuation. Northampton, MA: Edward Elgar. 
Merleau-Ponty, M. (1968). The Visible and the Invisible. Translated by Alphonso Lingis. Evanston III: Northwestern University Press.

Merleau-Ponty, M. (2006). El lenguaje indirecto y las voces del silencio. Buenos Aires: Nueva Visión.

Milstein, T. (2008). When whales "speak for themselves": Communication as a mediating force in wildlife tourism. Environmental Communication: A Journal of Nature and Culture, 2 (2), 173-192. https://doi. org/10.1080/17524030802141745

Milstein, T. (2011). Environmental communication theories. En: S.W. Littlejohn \& K. A. Foss (Eds.), Encyclopedia of communication theory (p. 344-349). Thousand Oaks, CA: Sage.

Milstein, T. \& Dickinson, E. (2012). Gynocentric greenwashing: The discursive gendering of nature. Communication, Culture, \& Critique, 5 (4), 510-532. https://doi.org/10.1111/j.1753-9137.2012.01144.x.

Milstein, T. \& Krolokke, C. (2012). Transcorporeal Tourism: Whales, fetuses, and the rupturing and reinscribing of cultural constraints. Environmental Communication: A Journal of Nature and Culture, 6 (1), 82-100. https://doi. org/10.1080/17524032.2011.642079

Nixon, R. (2011a). Slow violence and the environmentalism of the poor. Cambridge: Harvard University Press.

Nixon, R. (2011b). Slow Violence, Gender, and the Environmentalism of the Poor. En: B. Caminero-Santangelo, \& G. Myers (Eds.), Environment at the Margins: Literary and Environmental Studies in Africa, (pp. 257-285). Athens, OH: Ohio UP.

Packwood, C., Bekoff, M. \& Bexell, S. (2011). Giving voice to the "voiceless." Journalism Studies, 12 (5), 590-607. https://doi.org/10.1080/146167 OX.2010.540136 
Palacio, G. (2001). En búsqueda de conceptos para una historiografía ambiental. (pp. 36-73). En: Naturaleza en Disputa. Ensayos de Historia Ambiental de Colombia 1850-1995. Bogotá: Universidad Nacional de Colombia, Colciencias, Icanh.

Pérez-Marín, M. (2012). Discursos Ambientales: Una mirada histórica a la configuración del territorio del PNN Los Katíos en Colombia y su zona de amortiguación. Investigación y Desarrollo, 20 (2), 416-449.

Pérez-Marín, M. (2015a). Discursos Ambientales en Colombia: una mirada a los conflictos ambientales en el PNN Los Katíos. Propuesta de un proyecto investigativo eco-crítico postcolonial (Tesis de Doctorado). Doctorado en Ciencias Sociales, Universidad de Antioquia. Medellín, Colombia.

Pérez-Marín, M. (2015b). Representaciones sobre la Naturaleza y el Desarrollo en El Colombiano (1951-2010). En: Culturas, ecología humana y ciudadanías, pp. 116-132. Grupo de investigación Medio Ambiente y Sociedad - MASO, Universidad de Antioquia.

Pérez-Marín, M. (2016a). El discurso ambiental en Colombia: una mirada desde el análisis crítico del discurso. Chasqui. Revista Latinoamericana de Comunicación. 131, 131-139.

Pérez-Marín, M. (2016b). Critical Discourse Analysis of Colombian Identities and humanature in National Geographic Magazine (1903-1952) (Doctoral Dissertation). Recuperado de https://digitalrepository.unm.edu/cj_etds/1/

Pérez-Marín, M. (2017). Análisis Crítico del Discurso de la Identidad de los Colombianos en National Geographic Magazine (1903-1926). Signo y Pensamiento, 36 (71), 136-154.

Peterson, N.; Peterson, M. \& Peterson, T. (2007). Environmental Communication: Why the crisis discipline should facilitate environmental democracy. Environmental Communication: A Journal of Nature and Culture, 1, 74-86. https://doi.org/10.1080/17524030701334292 
Pezzullo, P. (2001). Performing critical interruptions: Stories, rhetorical invention, and the Environmental Justice movement. Western Journal of Communication,65(1), 1-25. https://doi.org/10.1080/10570310109374689

Pinchot, G. (1910). The Fight for Conservation, Nueva York: Doubleday, Page \& Co.

Ramírez, C. (1994). Hombre, Sociedad, educación y gobierno. San José: UNED

Ramos, J. (2002).Un precedente lejano del debate sobre sostenibilidad: el movimiento conservacionista americano. (1890-1920). ICE, Desarrollo Sostenible, (80), 31-46. Recuperado de http://www.revistasice.com/ CachePDF/ICE_800_31-46_D6C0C94FEB041B50816C572FEECBDDCF. pdf

Rappaport, J. (1990). The Politics of Memory. Cambridge: Cambridge University Press.

Resumen Operación Milagro II. (3 de mayo, 2016). Recuperado de https:// www.youtube.com/watch?v=xTkgKh07qyg

Rogers, R. (1998). Overcoming the objectification of nature in constitutive theories: Toward a transhuman, materialist theory of communication. Western Journal of Communication, 62, 244-272. https://doi. org/10.1080/10570319809374610

Rojas, C. (2002). Civilization and violence: Regimes of representation in nineteenth century Colombia. Minneapolis: University of Minnesota.

Rojas Caldelas, R.I. (2011). ¿Existe planeación sustentable de los asentamientos rurales? El caso de Baja California. ACE: Architecture, City and Environment, 6 (16), 129-144. Recuperado de https://upcommons.upc. edu/handle/2099/10535 
Rummenhoeller. K. (1995). Territorios indígenas y áreas naturales protegidas. (pp. 65-90). En: Tierra Profana: Grandes proyectos en territorios indígenas de Colombia. Bogotá: Unión Europea y Disloque Editores.

Stibbe, A. (2012). Animals erased: Discourse, ecology, and reconnection with the natural world. Middletown, CT: Wesleyan University Press.

Suárez, A. (2017). Mayor participación en políticas de pesca, proponen OSC y pescadores en Foro de Consulta Regional para la Pesca Sustentable. Environmetnal Defense Fund de Mexico. Recuperado de http://mexico. edf.org/prensa/mayor-participacion-en-politicas-de-pesca-proponen-oscy-pescadores-en-foro-de-consulta

Taylor, D. (2000). The rise of the environmental justice paradigm: Injustice framing and the social construction of environmental discourses. American Behavioral Scientist, 43 (4), 508-580. https://doi.org/10.1177/0002764200043004003

Tiburcio-Pintos, G. y Cariño-Olvera, M.M. (2017). Esfuerzos colectivos para la conservación de las tortugas marinas en el Golfo de California. Letras Verdes, (22), 7-26. https://doi.org/10.17141/letrasverdes.22.2017.2697

Tinker, G. (1996). An American Indian theological response to ecojustice. En: J. Weaver (Eds.), Defending Mother Earth: Native American Perspectives on Environmental Justice (pp. 153-176). Maryknoll, N.Y.: Orbis Books.

Valenzuela Reyes, M.D. (2012). Las cooperativas de producción pesquera. Su lugar en la Constitución y en las diversas leyes de la pesca mexicana. Letras Jurídicas, 26. 137-158. Recuperado de http://letrasjuridicas.com.mx/ Volumenes/26/A9.pdf

Valladolid, J. \& Apffel-Marglin, F. (2001). Andean cosmovision and the nurturing of biodiversity. In J.A. Grim (Eds.), Indigenous Traditions and Ecology: The Interbeing of Cosmology and Community (pp. 639-670). Cambridge, MA: Harvard Press. 
Vance, E. (2017, septiembre). Mayordomos del Mar. National Geographic en Español, 41 (3), 18-37.

Como citar: Pérez-Marín, M., y Cruz Hernández, S. (2020). Identidades ecoculturales en disputa: una mirada a los conflictos ambientales relacionados con la pesca en México. Revista KEPES, 17 (21), 289-333. DOI: 10.17151/kepes.2020.17.21.11

\section{Anexos}

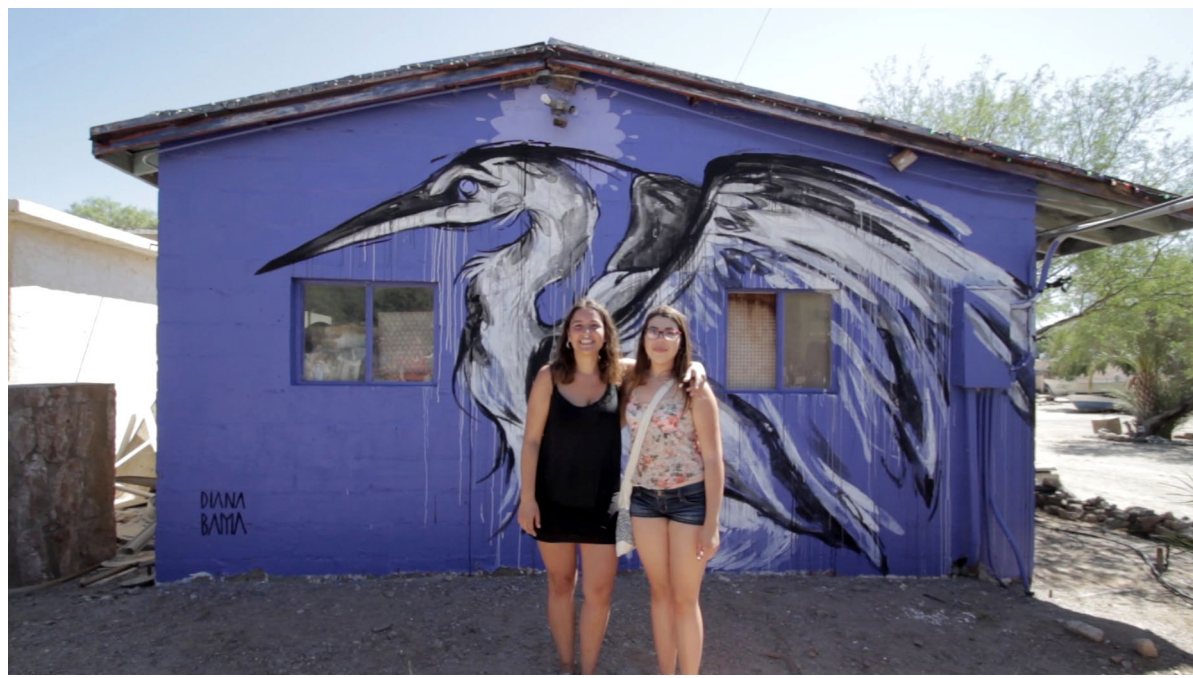

Mural 1. Imagen Mural "Garza"

Mural realizado por Diana Bama, artista de origen español. Está ubicado en el lugar del poblado llamado Guillermós. Fotografía cortesía de Adrián Macías y Marco Meza (2017). 


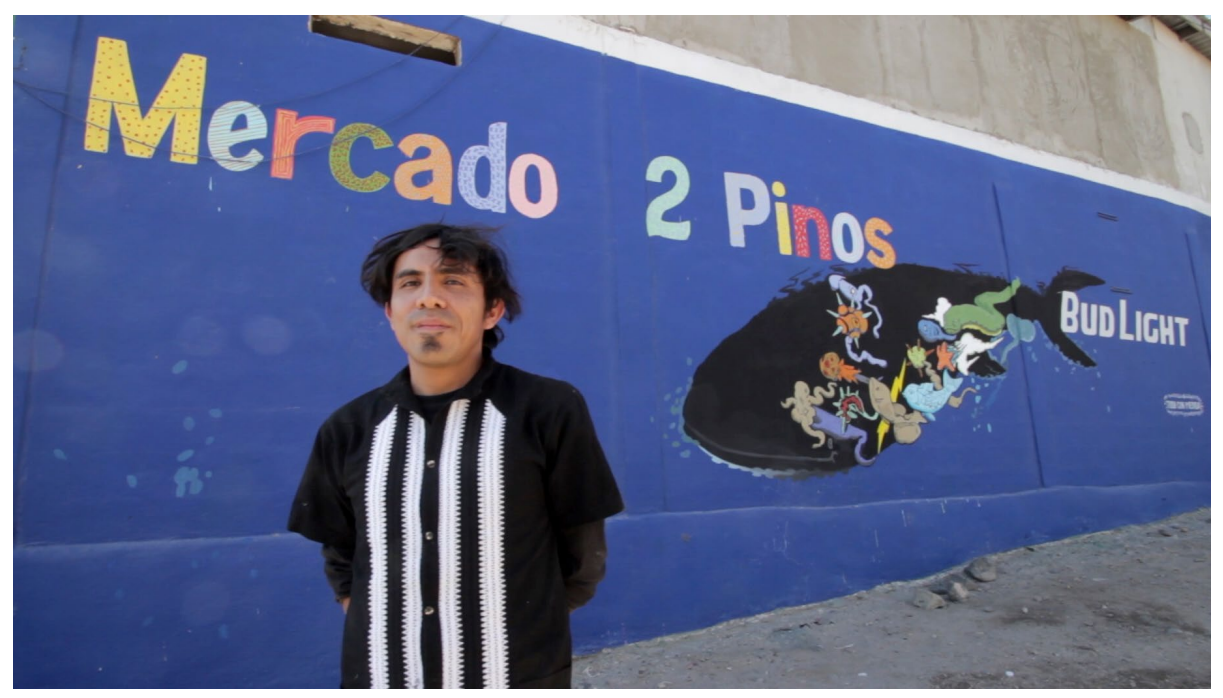

Mural 2. Imagen Mural “Personajes de Bahía de los Ángeles".

Mural realizado por Emerson Balderas, artista mexicano residente de Tlaxcala. Está ubicado en Mercado Los Pinos. Fotografía cortesía de Adrián Macías y Marco Meza (2017). 
Pérez-Marín, M., y Cruz Hernández, S.. / Identidades ecoculturales en disputa:

Una mirada a los conflictos ambientales relacionados con la pesca en México.

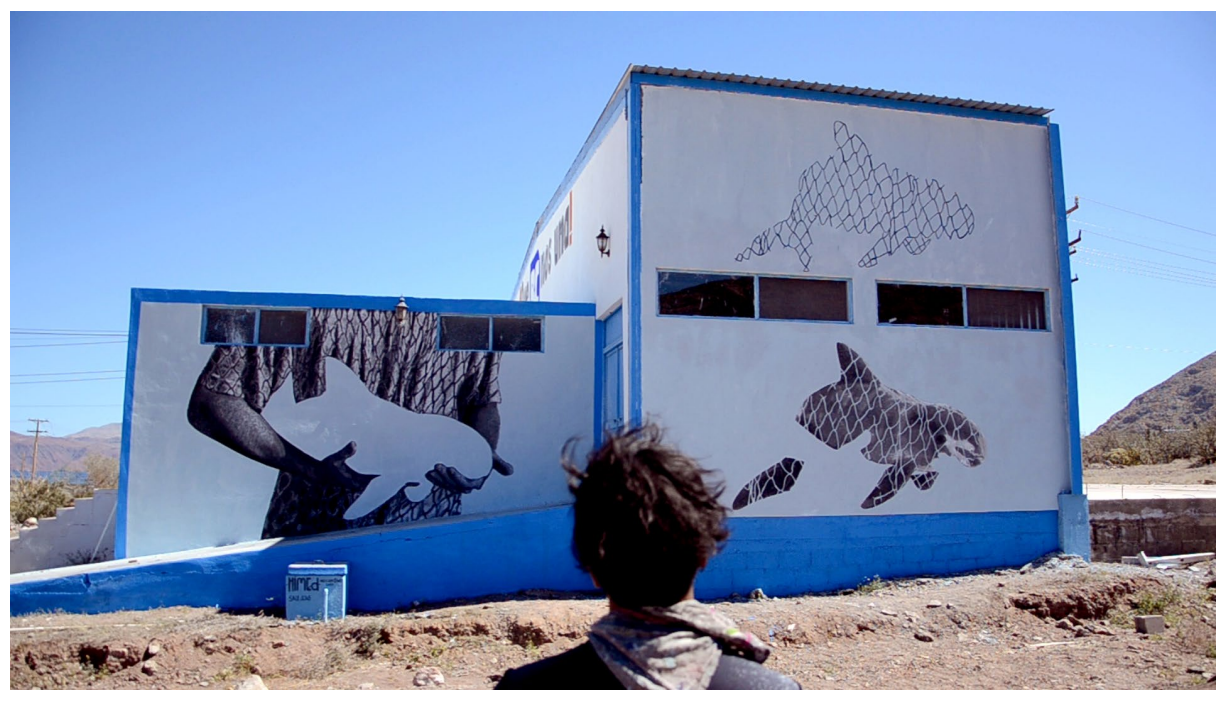

Mural 3. Imagen Mural "Vaquita Marina".

Mural realizado por Hymed, artista originario de Querétaro. Está ubicado en Casa de la cultura del Instituto de Cultura de Baja California. Fotografía cortesía de Adrián Macías y Marco Meza (2017) 


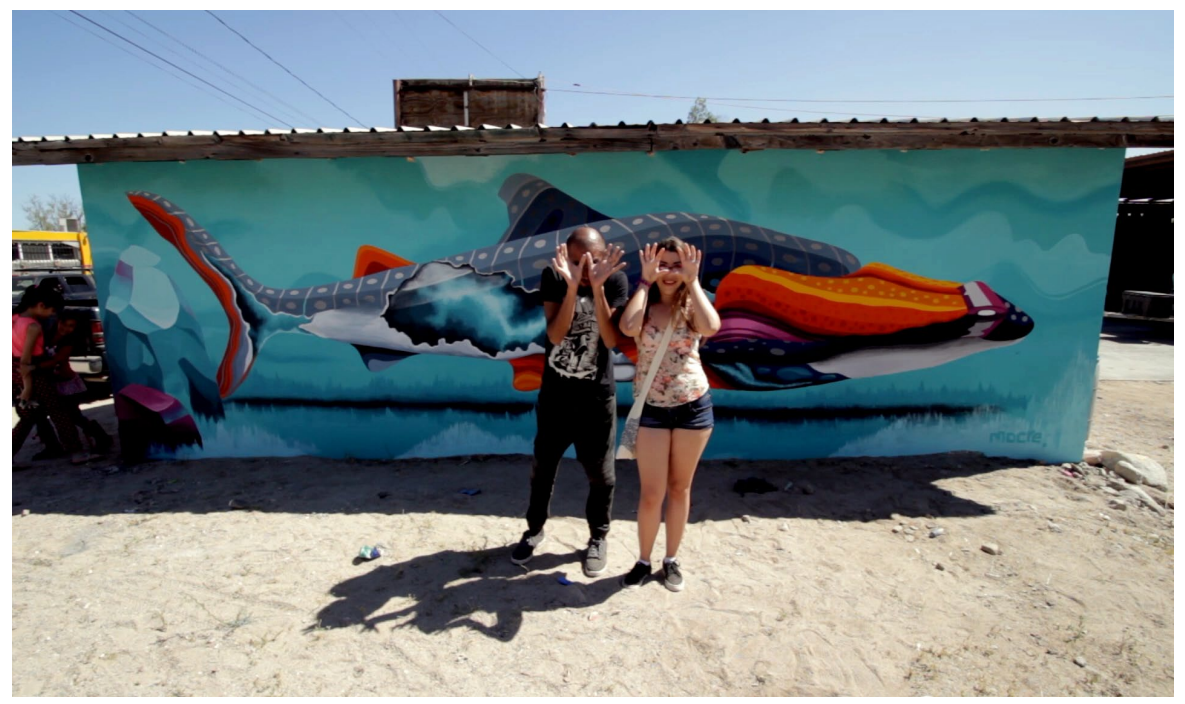

Mural 4. Imagen Mural "Tiburón Ballena"

Mural realizado por Mocre, artista originario de la ciudad de México. Está ubicado al frente del Mercado Xitlalli. Fotografía cortesía de Adrián Macías y Marco Meza (2017). 
Pérez-Marín, M., y Cruz Hernández, S.. / Identidades ecoculturales en disputa:

Una mirada a los conflictos ambientales relacionados con la pesca en México.

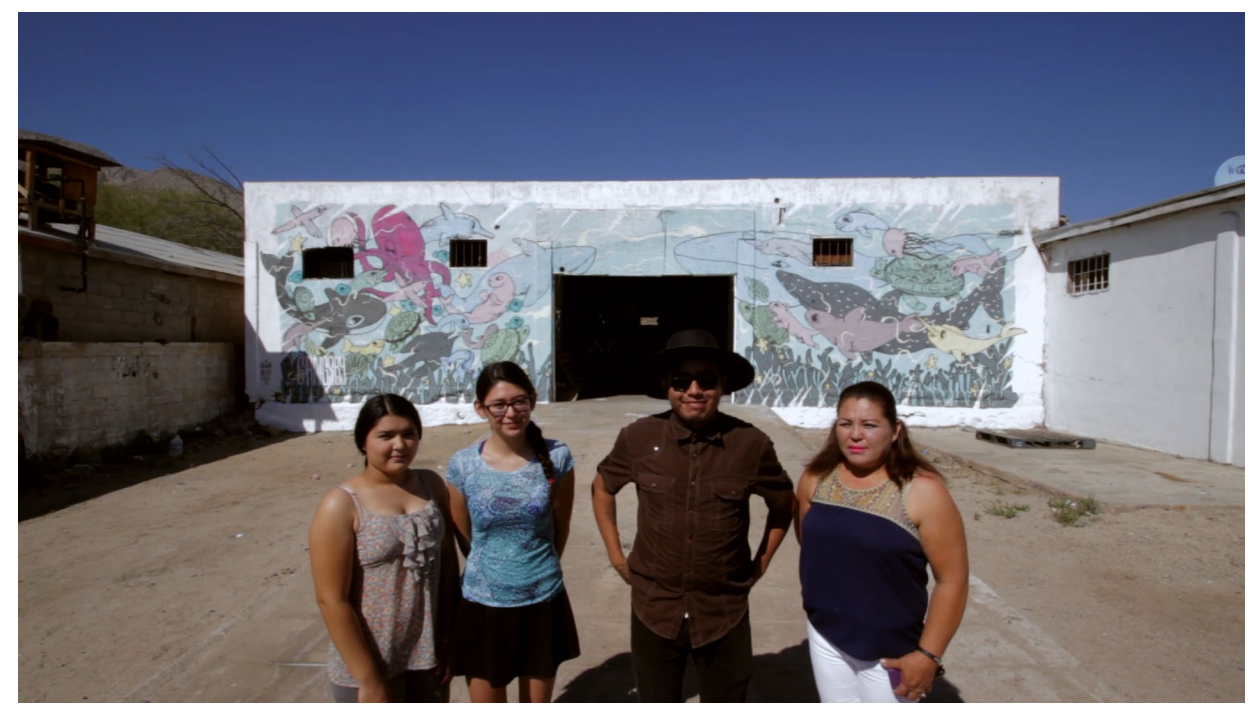

Mural 5. Imagen Mural "El rey tortuga"

Mural realizado por el artista mexicano Gangsby Davs. Está ubicado en el Salón de eventos del poblado. Fotografía cortesía de Adrián Macías y Marco Meza (2017). 


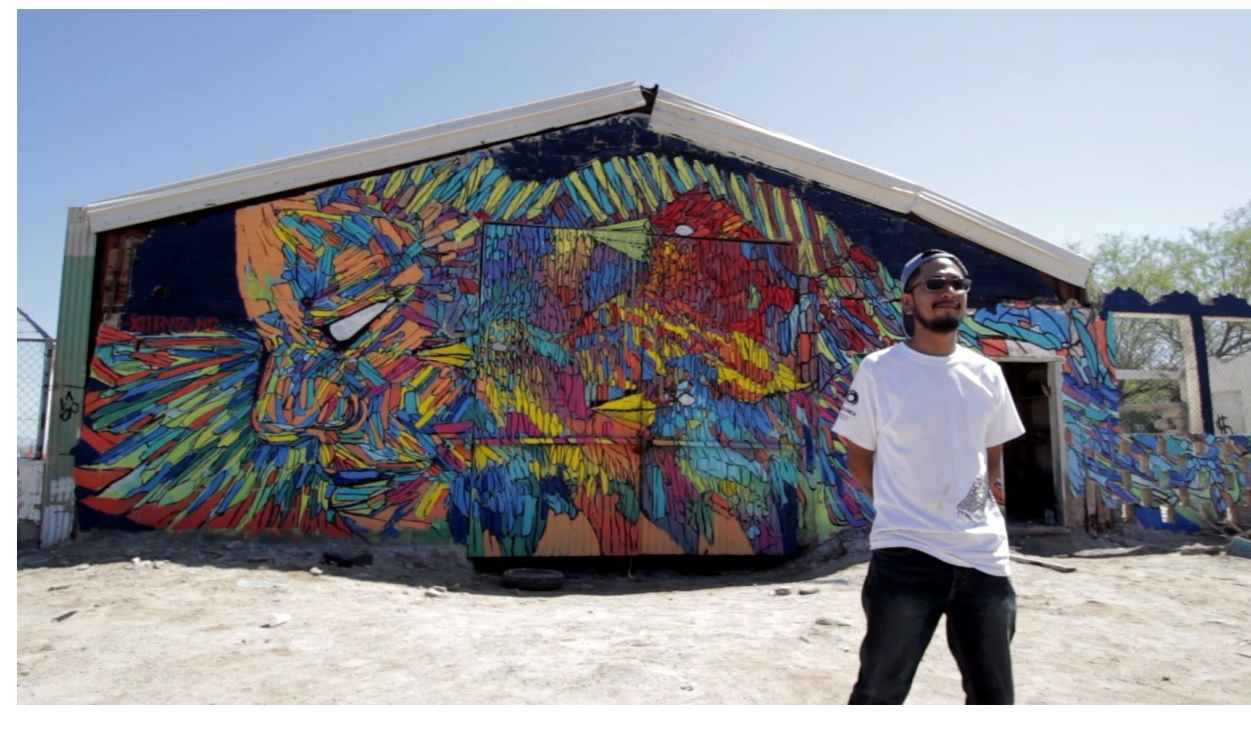

Mural 6. Imagen Mural "Vitral"

Mural realizado por Carlos Soto Xchams, artista mexicano. Está ubicado en la Casa Díaz. Fotografía cortesía de Adrián Macías y Marco Meza (2017). 
Pérez-Marín, M., y Cruz Hernández, S.. / Identidades ecoculturales en disputa:

Una mirada a los conflictos ambientales relacionados con la pesca en México.

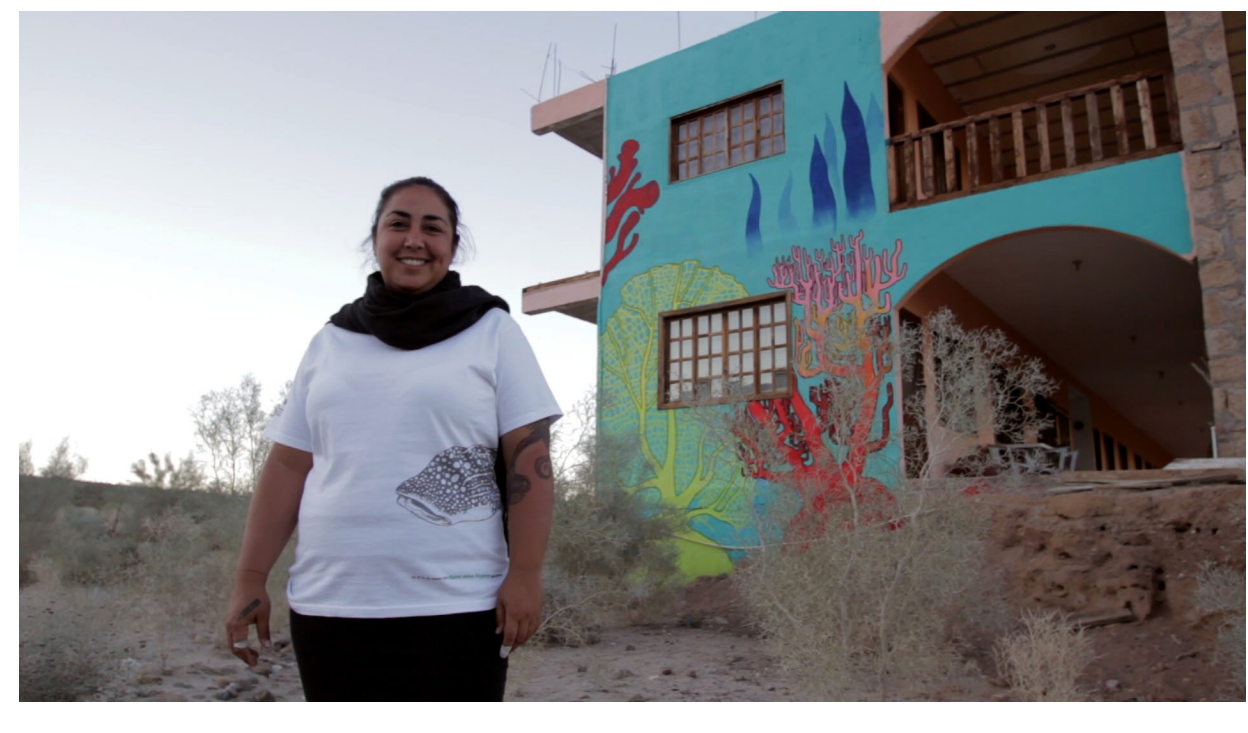

Mural 7. Imagen Mural "Corales"

Mural realizado por Esther Gámez, artista radicada en Ensenada integrante de Cardumen quienes organizaron el Festival de Arte Público. Está ubicado en el Hotel Los Corales.

Fotografía cortesía de Adrián Macías y Marco Meza (2017). 


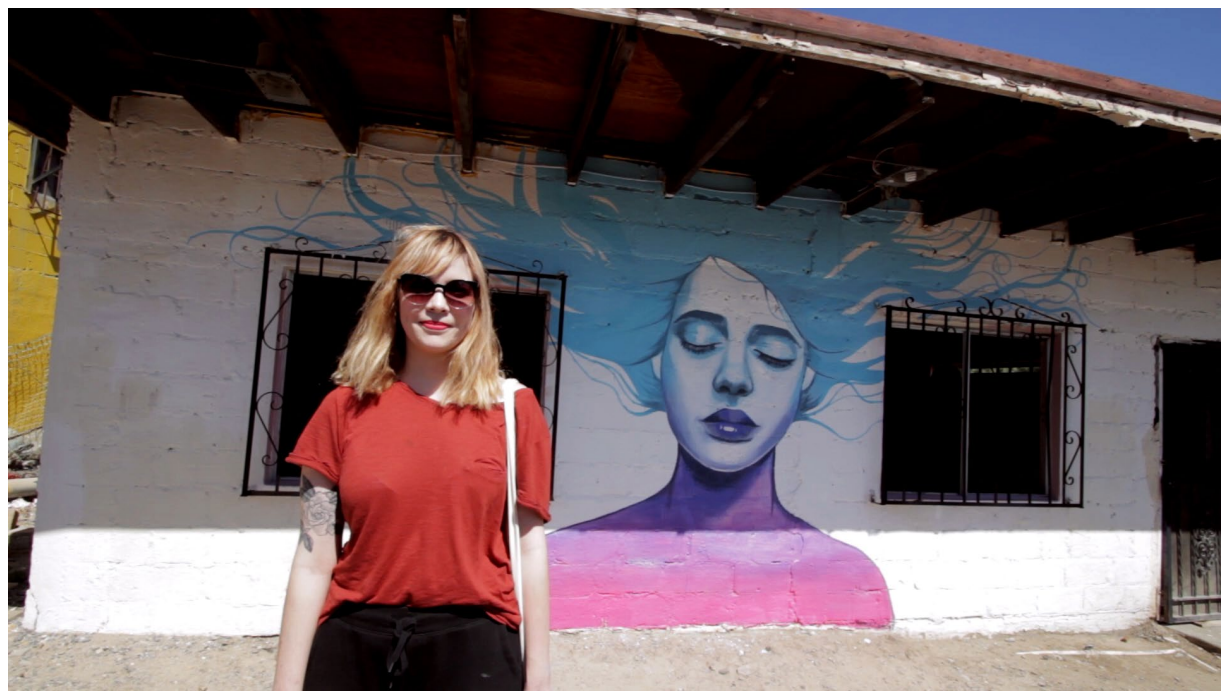

Mural 8. Imagen Mural "Mujer del mar"

Mural realizado por Mónica Loya, artista originaria de Chihuahua. Está ubicado en Casa frente a oficina Pronatura Noroeste, A.C. Fotografía cortesía de Adrián Macías y Marco Meza (2017) 
Pérez-Marín, M., y Cruz Hernández, S. . / Identidades ecoculturales en disputa:

Una mirada a los conflictos ambientales relacionados con la pesca en México.

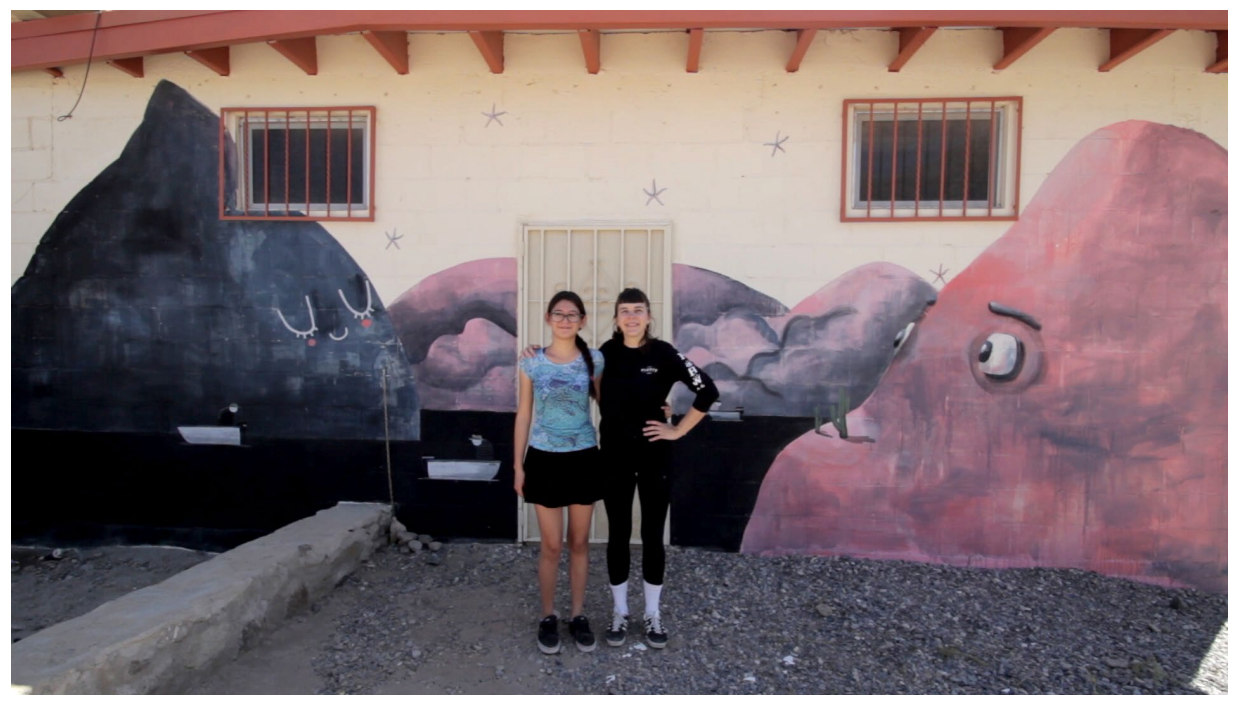

Mural 9. Imagen mural "Cerros e islas coloreados por el atardecer".

Mural realizado por la artista canadiense Cyrielle Tremblay. Está ubicado en el Museo de Historia Natural. Fotografía cortesía de Adrián Macías y Marco Meza (2017). 


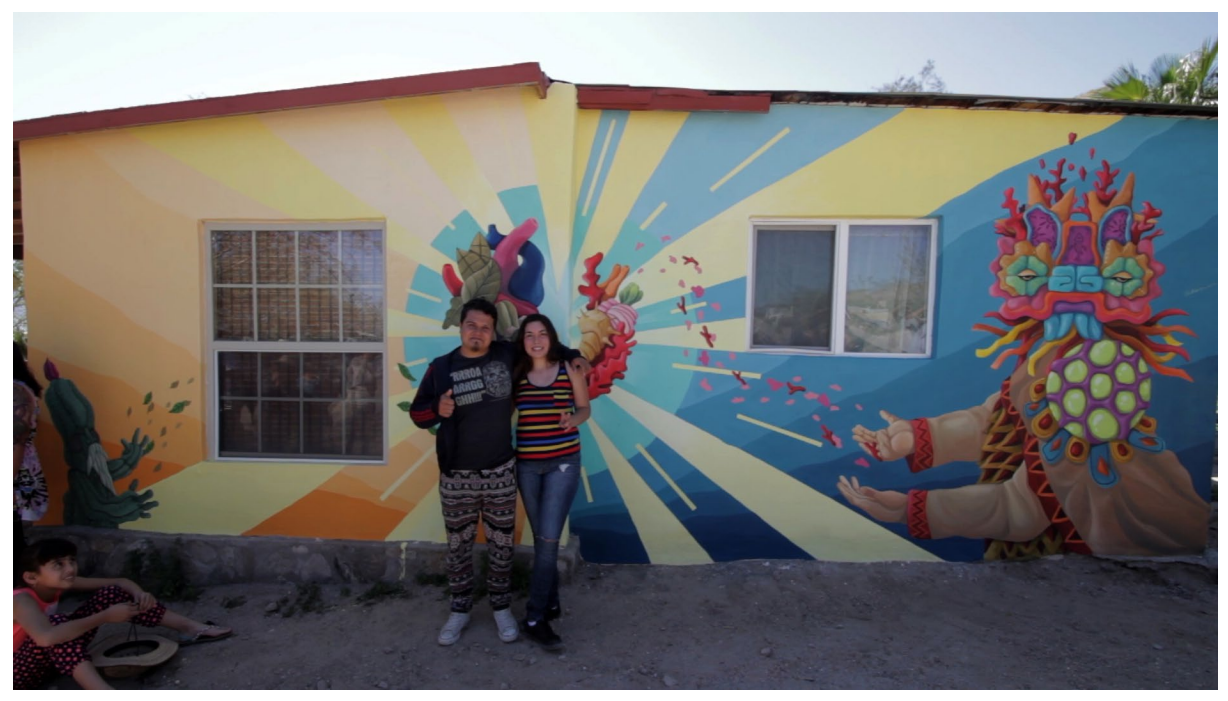

Mural 10. Imagen Mural "Corazón de mar y tierra”.

Mural realizado por el artista Dherzú Uzala de Cancún Quintana Roo. Está ubicado en la Casa Jana Chee. Fotografía cortesía de Adrián Macías y Marco Meza (2017). 
Pérez-Marín, M., y Cruz Hernández, S.. / Identidades ecoculturales en disputa:

Una mirada a los conflictos ambientales relacionados con la pesca en México.

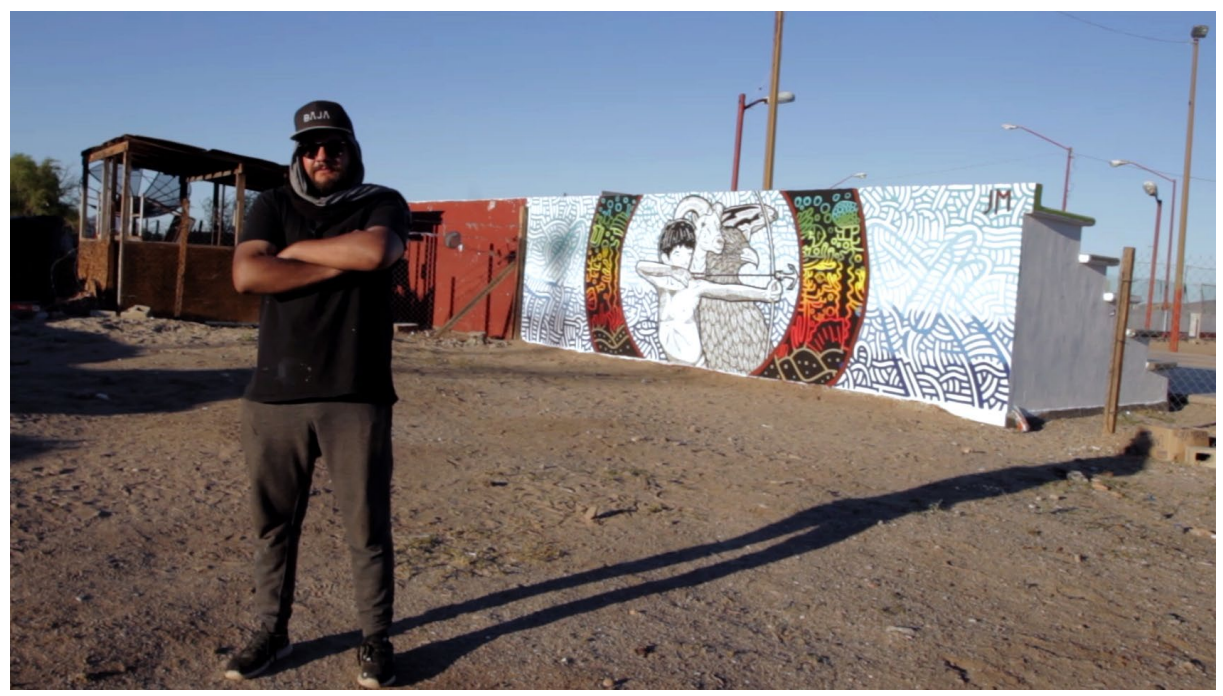

Mural 11. Imagen Mural "Niño Kiliwa".

Mural realizado por el artista bajacaliforniano, originario de Mexicali, JM. Está ubicado en la pared de las canchas atrás del Mercado La Isla. Fotografía cortesía de Adrián Macías y Marco Meza (2017). 